\title{
ORAL CARCINOGENESIS AND ROLE OF BACTERIA
}

\author{
SHAH R ${ }^{1}$, TALUKDAR DC ${ }^{2}$, FAKIR MAY $^{3}$, KHAN AFM $^{4}$, DATTA R ${ }^{5}$, SHAH TA ${ }^{6}$
}

\begin{abstract}
:
"Cancer", till date stands rigidly unconquered despite most strenuous effort and relentless endeavor by the scientists around the world. In global terms Oral cancer is among the most common malignancy and represents one of the greatest management challenges for the otolaryngologists as well as by the head and neck surgical oncologists.

Besides accumulation of genetic mutations, numerous other carcinogens including viral and chemical are well studied and documented. However, in the oral cavity, the role of microbiota in carcinogenesis is not known. Microbial populations on mouth mucosa differ between healthy and malignant sites, and certain oral bacterial species have been linked with malignancies, but the evidence is still weak in this respect. Nevertheless, oral microorganisms inevitably upregulate cytokines and other inflammatory mediators that affect the complex metabolic pathways, and may thus be involved in carcinogenesis. Poor oral health also associates statistically with prevalence of many types of cancer such as pancreatic and gastrointestinal cancer. This review presents commonly implicated bacteria in oral carcinogenesis, and their role in cancer therapeutics as well.
\end{abstract}

Key words: Bacterial carcinogenesis, carcinogenesis, Streptococcus anginosus

J Dhaka Med Coll. 2013; 22(2) : 211-215.

\section{Introduction:}

Head and neck carcinomas are biological heterogeneous group of cancers, in which oral cancer is the most common neoplasm. It is sixth common malignancy. Ninety percent of oral cancers are squamous cell carcinomas originating from the mucosal epithelium. They are the major cause of cancer morbidity and mortality worldwide, especially in the Indian subcontinent. ${ }^{1,2}$

As far as oral cancer is considered, $70-80 \%$ of oral cancer has been majorly linked to betel quid, tobacco chewing, smoking, and alcohol consumption. Other factors like genetic susceptibility of the individual and external agents such as dietary factor may exert their synergistic role in tumor genesis. ${ }^{1}$

The most common genetic alteration in oral cancer is mutation in tumor suppressor gene TP53 and retinoblastoma gene $(R b)$.
These mutations may be due to genetic alteration as in Li Fraumeni Syndrome or the effect of chemical carcinogenic agents and viral agents. Among the viral agents, association of human papilloma virus (HPV) is well documented. ${ }^{1,2,3}$

In the past decade, there has been increasing interest on the possible relationships between bacteria and the different stages of cancer development, but the association of bacteria with cancer of the oral cavity has yet to be adequately examined. In this review, we discussed the relationship of bacteria and their role in different stages of cancer development.

\section{Bacteria and oral cancer:}

Recently, it has been reported that the Streptococcus anginosus DNA sequence was found in DNA samples extracted from esophageal cancers. Because smoking and alcohol abuse are regarded as risk factors for

1. Dr. Rameez Shah, Dept of ENT \& Head Neck Surgery, Dhaka Medical College Hospital, Dhaka.

2. Dr. Debesh Chandra Talukdar, Assistant Professor, Dept of ENT \& Head Neck Surgery, Dhaka Medical College, Dhaka.

3. Dr. Md. Abu Yousuf Fakir, Associate Professor, Dept of ENT \& Head Neck Surgery, Dhaka Medical College, Dhaka.

4. Prof. A. F. Mohiuddin Khan, Professor and Head, Dept of ENT \& Head Neck Surgery, Dhaka Medical College, Dhaka.

5. Dr. Raj Datta, Resident Physician, Dhaka Medical College

6. Dr. Tazin Afrose Shah, Associate Professor of Medicine, Central International Medical College, Dhaka Correspondence: Dr. Rameez Shah, Dept of ENT \& Head Neck Surgery, Dhaka Medical College Hospital, Dhaka, Email: rameezshah527@yahoo.com 
both esophageal cancer and head and neck cancer, infection of $S$. anginosus might be associated with carcinogenesis of head and neck cancer. ${ }^{5}$

To investigate the involvement of $S$. anginosus infection in head and neck cancer, Tateda et al analyzed 217 DNA samples prepared from head and neck squamous cell carcinomas. By polymerase chain reaction (PCR) analysis, the $S$. anginosus DNA sequence was found in 217 out of 217 (100\%) DNA samples obtained from head and neck cancers. By Southern blot analysis, positive bands were detected in 41 out of 125 (33\%) samples. They could find no $S$. anginosus colony in oropharyngeal bacteriological culture dishes of 53 patients with and without head and neck cancer. On the other hand, they found the $S$. anginosus DNA fragment in eight out of eight DNA samples obtained from gingival smears by PCR analysis. These data indicate that the upper aerodigestive environment of the patients permitting $S$. anginosus infection is implicated in the carcinogenesis of head and neck squamous cell carcinoma. ${ }^{5}$

A hospital-based, case-control study was conducted on 20 patients with newly diagnosed oral cancer and 20 healthy controls without any cancer to evaluate the associations between $H$. pylori infection and oral cancer using culture and 16sRNA PCR technique for bacterial identification. However, the results of the pilot study suggest a possible association of $H$. pylori with an increased risk of oral cancer. Anand et al suggested additional studies in larger populations are necessary to confirm and quantify this possible association more accurately. ${ }^{6}$

Betel chewing has been shown to predispose to periodontal disease and oral cancer. Studies show that people with gum disease are more likely to test positive for $H$. pylori. It is not known if the lesions produced by betel quid and the resulting chemical changes predispose to colonization byH. pylori. Further, the role of this organism in oral cancer is not known. Author undertook a study to determine the presence of $H$. pylori in oral lesions of 30 oral cancer patients and to determine the presence of IgG antibodies to $H$. pylori in oral cancer patients who are betel chewers, non-betel chewers, healthy betel chewers, and healthy non-betel chewers, and to compare the presence of $H$. pylori in these four groups. One hundred and seventy-three subjects, of whom 53 were patients presenting with oral cancer to the Cancer Institute Maharagama, 60 were healthy betel chewers, and 60 were healthy non-betel chewers from the Religious and Welfare Service Centre Maharagama, were tested for $H$. pylori by serology. Thirty oral biopsies from oral cancer patients were cultured under microaerophilic condition to isolate $H$. pylori. Fourteen $(26.4 \%)$ of the oral cancer patients tested positive for $H$. pylori by serology, of which two were also culture positive (only 30 samples were cultured). The presence of $H$. pylori in betel chewers (with or without cancer) compared to non-betel chewers was statistically significant (Chi-square test $P<0.05)$. The use of tobacco and areca nut in betel chewers was significant with the presence of $H$. pylori $(P<$ $0.05)$. The oral cavity has been considered a potential reservoir for $H$. pylori, from where the organism causes recurrent gastric infections. ${ }^{7}$

In a case-control study, Rajendra et al. ${ }^{8}$ concluded that the contribution of the $H$. pylori in dental plaque to mucosal inflammation and periodontal disease was significant. Logistic regression analysis showed gastrointestinal disease and poor oral hygiene as being the greatest risk factors for bacterial colonization, irrespective of the subject groups. A positive correlation exists between rapid urease test (RUT) reactivity and the frequency of mucosal inflammation. Further, they suggested the importance of sustained lymphocytic infiltration of the tissue reaction in oral sub mucous fibrosis, which is a premalignant condition. ${ }^{9}$

Mager et $\mathrm{al}^{10}$ investigated if the salivary counts of 40 common oral bacteria in subjects with an OSCC lesion would differ from those found in cancer-free (OSCC-free) controls. Unstimulated saliva samples were collected from 229 OSCCfree and 45 OSCC subjects and evaluated for their content of 40 common oral bacteria using checkerboard DNA-DNA hybridization. They 
concluded that high salivary counts of Capnocytophaga gingivalis, Prevotella melaninogenica, and Streptococcus mitis may be diagnostic indicators of OSCC.

Hooper et al. in $2006^{11}$ conducted a study where, in order to characterize the bacterial microbiota present within oral cancerous lesions, tumorous and non-tumorous mucosal tissue specimens (approx. $1 \mathrm{~cm}^{3}$ ) were harvested from ten OSCC patients at the time of surgery. A total of 70 distinct taxa were detected and 52 different phylotypes were isolated from the tumorous tissues and 37 taxa from within the non-tumorous specimens. Differences between the composition of the microbiotas within the tumorous and nontumorous mucosae were apparent, possibly indicating selective growth of bacteria within carcinoma tissue. Most taxa isolated from within the tumor tissue represented saccharolytic and aciduric species. Whether the presence of these bacteria within the mucosa has any bearing on the carcinogenic process is a concept worthy of further investigation.

In 2007, Hooper et al. ${ }^{12}$ conducted a study with the primary objective to identify any bacterial species within OSCC tissue using a standard microbiological culture approach. At the time of surgery, a $1 \mathrm{~cm}^{3}$ portion of tissue was harvested from deep within the tumor mass using a fresh blade for each cut. Diverse bacterial taxa were isolated and identified, including several putatively novel species. Most isolates were found to be saccharolytic and acidtolerant species. Notably, some species were isolated only from either the tumorous or nontumorous tissue type, indicating a degree of restriction. Successful surface decontamination of the specimens indicates that the bacteria detected were from within the tissue. Diverse bacterial groups have been isolated from within OSCC tissue. The significance of these bacteria within the tumor warrants further study.

Jukka ${ }^{4}$ concluded in a review that microbial populations on mouth mucosa differ between healthy and malignant sites and certain oral bacterial species have been linked with malignancies, but the evidence is still weak in this respect. Nevertheless, oral microorganisms inevitably up-regulate cytokines and other inflammatory mediators that affect the complex metabolic pathways, and may thus be involved in carcinogenesis.

Sasaki et al. ${ }^{13}$ assessed the frequency of $S$. anginosus infection in oral cancer tissues and investigated its infection route. The tissue specimens were obtained from 46 oral cancer and 3 precancerous leukoplakia subjects. $S$. anginosus DNA was frequently detected in squamous cell carcinoma (19/42), but not in other types of cancer (lymphoma and rhabdomyosarcoma) or leukoplakia samples. A subject-based analysis revealed that $S$. anginosus was solely detected in dental plaque and not in saliva from all $19 \mathrm{~S}$. anginosuspositive squamous cell carcinoma cases. Further, the genotype of $S$. anginosus isolated from cancer tissue was identical to that from dental plaque of the same patients. They concluded that infection of $S$. anginosus could occur frequently in OSCC and that dental plaque could be a dominant reservoir of the $S$. anginosus.

Chocolatewala et al. ${ }^{1}$ concluded in a review that studies have shown diversity of isolated bacterial taxa between the oral cancer tissue specimens and the control, with Exiguobacterium oxidotolerans, P. melaninogenica, Staphylococcus aureus, and Veillonella parvula being specific for tumorigenic tissues. Most isolates are saccharolytic and acid tolerant. S. anginosus, commonly linked with esophageal and pharyngeal cancers, is not of significance in oral cancers. Similarly, significant salivary specificity is noted for three bacteria, namely, $C$. gingivalis, $P$. melaninogenica, and $S$. mitis, in oral cancer patients, making these species salivary markers for the early detection of oral cancers, and thus improving the survival rate significantly.

\section{Implication of bacteria in oral cancer therapeutics:}

The oral epithelium functions as a mechanical and protective barrier to resist bacterial infection. b-Defensins (BDs) are a group of antimicrobial peptides mainly produced by 
epithelial cells of many organs including skin, lung, kidney, pancreas, uterus, eye, and nasal and oral mucosa. This review focuses on BDs in oral epithelia and discusses their importance in oral epithelial health and disease. BDs exhibit antimicrobial activity against oral microbes including periodontitisrelated bacteria, Candida, and papilloma virus. Alterative expression of BDs was observed in oral epithelial diseases, including oral inflammatory lesions with and without microbial infection and oral cancer. BDs may be useful in the treatment of oral infectious diseases, ulcerative lesions, and cancer. BDs play an important role in protection against oral microbes and may be used in clinical applications. ${ }^{14}$

Azurin, a cupredoxin type of electron transfer and purified low-molecular-weight redox protein from the pathogenic bacteria Pseudomonas aeruginosa, selectively induces and triggers apoptosis in certain human cancer cells. ${ }^{[15]}$ Recently, Yamada et al. ${ }^{[16]}$ reported that azurin can effectively enter human cancer cells but not normal cells. After internalization, azurin forms a complex with the tumor suppressor protein p53 and stabilizes it, ${ }^{[17]}$ thereby inducing apoptosis or cell cycle arrest in the G1 phase. [18],[19] Despite extensive analysis of azurin's antitumor activity, its ability to modulate oral cancer growth has not yet been characterized. In this study, the antitumor effect of azurin on YD-9 and MG-63 cells is elucidated. Azurin controls p53 and cyclin B1 protein levels, leading to apoptosis of OSCC. Furthermore, combination treatment of azurin with 5-fluorouracil or etoposide effectively increases the sensitivity of OSCC to anticancer drugs. ${ }^{15}$

Increasing epidemiological evidence supports the view that dietary flavonoids have protective roles in oral diseases, including cancer. However, the dietary forms of flavonoids, the flavonoid glycosides, must first be hydrolyzed to the aglycones, which is thought to occur mainly in the intestine. Author tested whether this hydrolytic activity occurs in the oral cavity. Their evidence supported the contention that salivary hydrolysis of certain flavonoid glucosides may be important in some individuals but not in others. Support for a bacterial contribution to this hydrolysis was obtained from the inhibitory effect of antibacterials in vivo and in vitro and from experiments with subcultured oral bacterial colonies. However, cytosol isolated from oral epithelial cells was also capable of effective hydrolysis. Dietary flavonoid glucosides may thus be hydrolyzed in the oral cavity by both bacteria and epithelial cells that are shed, to deliver the biologically active aglycones at the surface of the epithelial cells. The aglycones quercetin and genistein both potently inhibited proliferation of oral cancer cells. The large interindividual variability in this hydrolytic activity may be a factor that should be taken into consideration in future studies. ${ }^{20}$

Comprehensive comparison of the salivary microbiota between patients with pancreatic cancer and healthy control subjects revealed a significant variation of salivary microflora. The authors observed associations between variations of patients' salivary microbiota with pancreatic cancer and chronic pancreatitis. This report also provides proof of salivary microbiota as an informative source for discovering non-invasive biomarkers of systemic diseases. $^{21}$

\section{Conclusion:}

Studies on bacterial carcinogenesis are lacking; unlike HPV, direct link of bacteria to oral cancer cannot be proved by available literature. Yet, there is a significant difference in oral bacteria in cancerous and noncancerous tissues. We can hypothesize that like $\mathrm{HBV}$ and $\mathrm{HCV}$, bacteria can maintain inflammation in surrounding stroma by induction of certain inflammatory cytokines, which have a role in carcinogenesis and invasion. Furthermore, research in the the role of bacteria in induction of tumor-induced macrophage which has immunosu prresive effect on the tumor, will throw more light in to bacterial carcinogenesis Prognosis of bacterial induced oral cancer may be good because this can be easily counteracted by antimicrobials and induction of certain beneficial biological reactions in cancer tissue, as mentioned in treatment aspect of the present review. 


\section{References:}

1. Chocolatewala N, Chaturvedi P, Desale R. The role of bacteria in oral cancer. Indian $\mathrm{J}$ Med Paediatr Oncol 2010; 31: 126-31.

2. Chocolatewala NM, Chaturvedi P. Role of human papilloma virus in the oral carcinogenesis: An Indian perspective. J Cancer Res Ther 2009; 5: 71-7.

3. Vinay K, Nelso F, Abul A, Philadelphia. Pathological basis of disease. 7 th ed. W.B Sunders; 2010: p.269-342.

4. Meurman JH. Oral microbiota and cancer. J Oral Microbiol 2010; 2: 5195.

5. Tateda M, Shiga K, Saijo S, Sone M, Hori T, Yokoyama J, et al. Streptococcus anginosus in head and neck squamous cell carcinoma: Implication in carcinogenesis. Int $\mathrm{J}$ Mol Med 2000; 6: 699-703.

6. Anand D, Vineeta S, Mridula S, Royana S, Manoj P. Helicobacter pylori and oral cancer: possible association in a preliminary case control study. Asian Pac J Cancer Prev 2011; 12: 1333-6.

7. Fernando N, Jayakumar G, Perera N, Amarasingha I, Meedin F, Holton J. Presence of Helicobacter pylori in betel chewers and non betel chewers with and without oral cancers. BMC Oral Health 2009; 9: 23 .

8. Rajendran R, Rajeev R, Anil S, Alasqah M, Rabi AG. Helicobacter pylori coinfection is a confounder, modulating mucosal inflammation in oral submucous fibrosis. Indian J Dent Res 2009; 20: 206-11.

9. Rajendra R, Sivapathasundharam B. Shafer's text book of oral pathology. 5th ed. Gurgaon: Elsevier; 2007: p.37.

10. Mager DL, Haffajee AD, Devlin PM, Norris CM, Posner MR, Goodson JM. The salivary microbiota as a diagnostic indicator of oral cancer: A descriptive, non-randomized study of cancer-free and oral squamous cell carcinoma subjects. J Transl Med 2005; 3: 27.

11. Hooper SJ, Crean SJ, Lewis MA, Spratt DA, Wade WG, Wilson MJ. Viable bacteria present within oral squamous cell carcinoma tissue. J Clin Microbiol 2006; 44: 1719-25.
12. Hooper SJ, Crean SJ, Lewis MA, Spratt DA, Wade WG, Wilson MJ. A molecular analysis of the bacteria present within oral squamous cell carcinoma. J Med Microbiol 2007; 56: 1651-9.

13. Sasaki M, Yamaura C, Ohara-Nemoto Y, Tajika S, Kodama Y, Ohya T, et al. Streptococcus anginosus infection in oral cancer and its infection route. Oral Diseases 2005; 11: 151-6.

14. Yoshihiro A, Masto S, Michiko N, Mami Y, Daisuke S, Tohru K. Role of b-defensins in oral epithelial health and disease. Med Mol Morphol 2007; 40: 179-84.

15. Jeong-Hae C, Moo-Hyung L, Yun-Jung C, BongSoo P, Shin K, Gyoo-Cheon K. The bacterial protein azurin enhances sensitivity of oral squamous carcinoma cells to anticancer drugs. Yonsei Med J 2011; 52: 773-8.

16. Yamada T, Fialho AM, Punj V, Bratescu L, Gupta TK, Chakrabarty AM. Internalization of bacterial redox protein azurin in mammalian cells: Entry domain and specificity. Cell Microbiol 2005; 7 : 1418-31.

17. Yamada T, Goto M, Punj V, Zaborina O, Chen $\mathrm{ML}$, Kimbara K, et al. Bacterial redox protein azurin, tumor suppressor protein p53, and regression of cancer. Proc Natl Acad Sci 2002; 99: 14098-103.

18. Punj V, Bhattacharyya S, Saint-Dic D, Vasu C, Cunningham EA, Graves J, et al. Bacterial cupredoxin azurin as an inducer of apoptosis and regression in human breast cancer. Oncogene 2004; 23: 2367-78.

19. Yamada T, Goto M, Punj V, Zaborina O, Kimbara K, Das Gupta TK, et al. The bacterial redox protein azurin induces apoptosis in J774 macrophages through complex formation and stabilization of the tumor suppressor protein p53. Infect Immun 2002; 70: 7054-62.

20. Thomas W, Alyson MB, Lisa LS, Susan GR, Walle UK. Flavonoid glucosides; are hydrolyzed and thus activated in the oral cavity in humans. Hum Nutr Metabol 2005; 49-52.

21. Farrell JJ, Zhang L, Zhou H, Chia D, Elashoff D, Akin D, et al. Variations of oral microbiota are associated with pancreatic diseases including pancreatic cancer. Gut 2012; 61: 582-8. 